\title{
O USO DE PLANTAS MEDICINAIS DURANTE A GRAVIDEZ E AMAMENTAÇÃO
}

\section{MEDICINAL PLANTS DURING PREGNANCY AND BREASTFEEDING}

\section{Ana Flávia Schvabe DUARTE1; Antonio Luiz da Costa MARTINS²; Marilis Dallarmi MIGUEL ${ }^{3}$; Obdulio Gomes MIGUEL ${ }^{1}$}

1 - Universidade Federal do Paraná, Departamento de Farmácia, Laboratório de Fitoquímica, Curitiba, PR, Brasil.

2 - Hospital Santa Casa de Misericórdia de Curitiba, Curitiba, PR, Brasil.

3 - Universidade Federal do Paraná, Departamento de Farmácia, Laboratório de Farmacotécnica, Curitiba, PR, Brasil

Autor para correspondência: anaduarte.ufpr@gmail.com

\section{RESUMO:}

Parte dos fitoterápicos são consumidos sem acompanhamento por profissional de saúde, sendo de venda sem prescrição médica. Culturalmente, as gestantes optam pelo uso de plantas medicinais e fitoterápicos para tratarem desconfortos decorrentes ou não da gestação. Entretanto, na terapêutica, o uso de plantas medicinais com base e sendo assegurado somente pelo uso tradicional pode ser perigoso. É fundamental que se tenha um perfil tóxico bem estabelecido, principalmente no que se refere ao potencial abortivo e teratogênico, salientando que com relação as plantas medicinais as informações a esse respeito são escassas e contraditórias. Este estudo teve como objetivo realizar um levantamento bibliográfico com o propósito de apresentar questões relacionadas ao uso de plantas medicinais durante a gravidez e amamentação.

Palavras-chave: plantas medicinais, gestação, amamentação, toxicidade.

\section{ABSTRACT:}

Some herbal products are consumed without accompaniment of a health professional and sold without a prescription. Culturally, pregnant women opt for the use of herbal and phytotherapeutic plants to treat discomfort, whether or not they result from gestation. However, in therapeutics, the use of medicinal plants based on ,and being assured, only by traditional use can be dangerous. It is essential to have a well-established toxic profile, especially regarding the abortive and teratogenic potential, pointing out that in relation to medicinal plants the information in this respect is scarce and contradictory. The objective of this study was to carry out a bibliographic survey with the purpose of presenting questions related to the use of medicinal plants during pregnancy and breastfeeding.

Keyworks: medicinal plants, pregnancy, breastfeeding, toxicity.

\section{INTRODUÇÃO}

As plantas são parte integrante da cultura da civilização, utilizadas como fonte alimentar ou finalidade terapêutica. O uso de espécies vegetais com finalidade medicinal surgiu por meio de um método empírico tão antigo quanto a própria humanidade, auxiliando 
o processo de distinção entre as plantas próprias para o consumo daquelas que apresentavam toxicidade. Dessa maneira, as propriedades intrínsecas das plantas passaram a compor o repertório do conhecimento popular e cultural (MIGUEL, MIGUEL, 2000; DI STASI, 1996).

Com relação a importância de políticas públicas para o fortalecimento da fitoterapia no Sistema Único de Saúde (SUS), são marcos recentes a regulamentação da Política Nacional de Práticas Integrativas e Complementares no SUS (PNPIC) e a Política Nacional de Plantas Medicinais e Fitoterápicos (PNPMF) (BRASIL, 2006a; BRASIL, 2006b). No Brasil, os produtos fitoterápicos devem apresentar comprovação de eficácia e segurança, inclusive para uso na gravidez e lactação. Referente a legislação sobre a toxicidade reprodutiva de produtos fitoterápicos, tem-se a necessidade de avaliação sobre parâmetros de fertilidade, performance reprodutiva, efeitos adversos no decorrer da vida intra e extra uterina, efeitos adversos sobre a gestante, sobre o parto e o desenvolvimento pós-natal (BRASIL, 1996; BRASIL 2004).

O período gestacional é permeado por alterações inerentes a esse processo e que embora estejam dentro da normalidade, podem gerar queixas comuns de desconforto, como por exemplo náuseas, vômitos e constipação intestinal. Porém, o uso equivocado de plantas medicinais rotineiramente utilizadas pela população para o alívio das mais variadas afecções clínicas pode recair no paradigma de que o que é da natureza não faz mal, o que não é necessariamente verdade, visto que da natureza se extraem diversos compostos que apresentam toxicidade. Esse fato torna-se ainda mais preocupante quando o foco é a gestante, pois a busca pelo tratamento de uma afecção clínica por meio de uma determinada planta medicinal, pode ocasionar efeitos indesejados, seja pela indicação incorreta, ou mesmo pelo desconhecimento cultural. Entre os efeitos adversos que podem ocorrer devido ao uso incorreto de plantas medicinais e fitoterápicos pelas gestantes, encontram-se os efeitos teratogênicos e o aborto (CHEZ \& JONAS, 1997; ERNEST, 2002).

Considerando os aspectos descritos, este estudo reúne dados de literatura sobre o emprego de plantas medicinais durante a gestação e lactação, com o objetivo de alertar a população sobre esse tema.

\section{METODOLOGIA}

A pesquisa bibliográfica foi realizada nas bases de dados Medline, Scopus, Web of Science e Scielo utilizando os termos: pregnacy, lactation, toxicity, herbal medicines, 
phytomedicines. As plantas medicinais com uso mais difundido durante o período gestacional e de lactação, segundo as referências consultadas, foram selecionadas para pesquisa bibliográfica especifica, utilizando como descritores seus binômios científicos. Publicações de órgãos de regulamentação também foram utilizados.

\section{RESULTADOS}

\subsection{Sintomas comuns durante a gestação e plantas medicinais utilizadas}

\section{a) Náusea e vômito}

Durante a gestação, as náuseas e vômitos são queixas comuns. Esses sintomas geralmente iniciam entre a $4^{\circ}$ e $8^{\circ}$ semana de gestação e desaparecem por volta da $16^{\circ}$ semana. Sua gênese relaciona-se com altos índices do hormônio gonadotrófico humano, que pode apresentar altos índices em casos de gestação múltipla e doença trofoblástica gestacional. O hormônio gonadotrófico humano tem seu ápice no sangue materno por volta da $12^{\circ}$ semana de gestação, o que explica o fato da melhora desses sintomas após esse período. Porém, em aproximadamente $5 \%$ dos casos, esses episódios evoluem para uma condição grave, a hiperemese gravídica, causando desidratação, desnutrição, desequilíbrios hidroeletrolíticos e ácidos-básicos (MILLER, 2002; WOOLHOUSE, 2006; GOODWIN, 2002).

Zingiber officinale Roscoe, Zingiberaceae, conhecida popularmente como gengibre, é uma planta cujo rizoma tem largo emprego alimentar, industrial e medicinal (AFZAL et al., 2001; CHRUBASIK et al., 2005). Com relação às propriedades farmacológicas, o gengibre apresenta um amplo espectro de ação, destacando-se a atividade anti-inflamatória (FLYNN et al., 1968, GRZANNA et al., 2005; THOMSON et al., 2002; OJEWOLE, 2006), antimicrobiana (CHRUBASIK et al., 2005; FICKER et al., 2003, IQBAL et al., 2006) e seu uso no tratamento de distúrbios gastrointestinais. Especificamente com relação aos distúrbios gastrointestinais presentes durante o período gestacional, o gengibre demonstra ação farmacológica eficaz no tratamento de náuseas e vômitos. Revisões bibliográficas utilizando meta-análise de estudos clínicos randomizados (VILJOEN et al., 2014; THOMSON et al., 2014) abordam a questão do uso do gengibre durante a gravidez, utilizando diferentes dosagens (200 - $500 \mathrm{mg}$ ), esquemas posológicos, duração e grupos controle (placebo, vitamina B6, metoclopramida). Em geral, todos os experimentos 
demonstram ação estatisticamente semelhante a metoclopramida e a vitamina B6 na redução do enjoo, porém existem discordâncias com relação ao decréscimo do número de episódios de vômito. Com relação a toxicidade, Portnoi et al., (2003) demonstra os efeitos do uso do gengibre no primeiro trimestre de gravidez de 187 mulheres em comparação a aquelas que não foram expostas, observando que não houve diferença estatística entre os dois grupos com relação ao feto em termos de número de abortos espontâneos, número de natimortos, baixo peso ao nascer ou parto prematuro. Heitmann et al., (2013) demonstra em estudo de corte com 68.522 mulheres que o uso do gengibre durante a gravidez não foi associado com maior risco de malformação congênita, baixo peso ao nascer, parto prématuro e número de natimortos. Segundo Lete e Allué, (2016) o tratamento de náuseas e vômitos ocasionados pela gravidez utilizando o gengibre tem se mostrado uma alternativa de baixo custo e segura, não excluindo a necessidade de se realizarem mais estudos clínicos.

\section{b) Constipação}

Problemas de constipação são comuns em mulheres grávidas (JEWELL \& YOUNG, 2000). Esta queixa relaciona-se principalmente as alterações fisiológicas decorrentes da gravidez, como por exemplo a ação de hormônios específicos sobre a mobilidade intestinal (BARON et al., 1993).

É comum o uso de plantas que contem antraquinonas para o alivio da constipação. Porém, o uso dessas espécies deve ser visto com prudência, principalmente durante o primeiro trimestre de gravidez, pois entre as ações farmacológicas das antraquinonas temse indução de contrações uterinas, aumento de fluxo sanguíneo para o útero e anexos, com consequente risco de aborto. Com relação as espécies que contêm antraquinonas e que são comumente utilizadas com finalidade laxante, destacam-se: sene (Senna alexandrina Mill. Fabaceae), cáscara sagrada (Rhamnus purshiana DC. Rhamnaceae), frângula (Rhamnus frangula L., Rhamnaceae), ruibarbo (Rheum L., Polygonaceae) e aloe (Aloe L. Asphodelaceae (CONOVER, 2003; BELEW, 1999; SHULZ et al., 2002; FALKENBERG, 2010).

\subsection{Plantas medicinais galactagogas}


A amamentação é uma questão importante para a saúde humana, principalmente até os dois anos de vida, pois atende às necessidades nutricionais, metabólicas, imunológicas, além de proporcionar estímulo psicoafetivo. O leite materno é um alimento completo, atendendo as necessidades nutricionais do lactente nos primeiros seis meses de vida. Para o lactente, a amamentação apresenta benefícios no que se refere a prevenção de doenças, além de melhorar o desenvolvimento cognitivo. Com relação aos benefícios promovidos por essa prática, tem-se que a amamentação atua na prevenção da hemorragia pós-parto, promovendo a melhora da remineralização óssea com redução de fraturas do colo do fêmur no período pós menopausa, diminui o risco de câncer de mama e ovário e promove proteção contra o diabetes tipo 2 (NEWCOMB et al., 1994; REA et al., 2004; TOMA \& REA, 2008, CAMINHA et al., 2010).

A produção de leite materno origina-se a partir de uma complexa interação neuropsicoendócrina. Durante o período gestacional, o estrogênio e progestogênio exercem função no preparo das glândulas mamarias para a lactação. Apesar dos benefícios atribuídos a amamentação, são inúmeras as causas que levam a altas taxas de desmame. A amamentação exclusiva depende de um conjunto de fatores sociais, culturais e psicológicos, auxiliando na confiança e desejo da mãe em amamentar (MCNANAMAN \& NEVILLE,2003; GARTNER, 2005; FALEIROS et al., 2006).

Galactagogos são substâncias que auxiliam a iniciação, manutenção ou aumento da produção de leite. A indicação médica mais comum para o uso de galactagogos inclui mulheres com produção de leite insuficiente e que não respondem ao aconselhamento de profissional de saúde em lactação. Nesses casos, ocorre o aumento da produção de leite após doença ou separação materna e infantil e o reestabelecimento da produção de leite após o desmame. A terapia com uso de galactagogos pode ser indicada para induzir a lactação em mulheres que não deram a luz, como nos casos de adoção. Ao longo da história, as mulheres de diferentes culturas fazem uso de dietas alimentares e utilizam plantas com a intenção de aumentar a produção de leite (BETZOLD, 2004; TABARES et al., 2014).

Trigonella foenum graecum, Fabaceae, espécie conhecida popularmente como feno grego, possui uso alimentar e medicinal (BAHMANI et al., 2016). Com relação as atividades farmacológicas, demonstra atividade hipoglicemiante, antioxidante, gastroprotetora, hepatoprotetora e galactagoga. Contudo, o feno grego não deve ser utilizado por gestantes pois pode causar anomalias congênitas (ZAPANTIS et al., 2012; TALOUBI et al, 2013; OUZIR et al., 2016; GOYAL et al., 2016; VENKATA et al., 2017). 
Foeniculum vulgare, Apiaceae, conhecida popularmente como funcho, tem uso medicinal, alimentar e na cosmética. Apresenta atividade antimicrobiana, antiviral, antioxidante, anti-inflamatória, antialérgica, antinociceptiva e galactagoga (BADGUJAR et al., 2014; TABARES et al., 2014; RAHIMI \& ARDEKANI, 2013). Foeniculum vulgare não deve ser utilizado por gestantes, pois apresenta potencial teratogênico (BARILLI et al., 2012).

\subsection{Plantas medicinais contraindicadas durante a gestação}

A gestação é uma experiência singular, onde a exposição a uma determinada substância afeta dois organismos. A resposta do feto difere da resposta fisiológica observada na mãe, podendo ocasionar toxicidade embrionária e fetal. Salvo raras exceções, uma droga que exerça um efeito sistêmico na gestante atravessa a placenta e chega ao feto. Portanto, durante a gestação, recomenda-se evitar qualquer medicação, principalmente durante o primeiro trimestre, salvo indicação para sua utilização. No entanto, o uso de medicamentos durante a gestação é uma situação frequente, considerando que a condição de gestante não exclui a possibilidade da necessidade do tratamento de doenças crônicas ou intercorrentes (PALMER, 1981; RUEDY, 1984; MENGUE et al., 2001).

Com relação as plantas medicinais e medicamentos fitoterápicos, uma parcela da população acredita que seu emprego constitui parte do saber popular, sendo seguro, não gerando efeitos adversos e nem interação com outros medicamentos (LOUIK et al., 2010). Partindo desses princípios, é comum a prática da auto-medicação, muitas vezes sem informar ao profissional de saúde quando questionado sobre o uso de medicamentos. $O$ reino vegetal é uma reconhecida fonte de plantas com propriedades medicinais, utilizadas na terapêutica e popularmente para o reestabelecimento de desordens fisiológicas, sendo ainda cultural e milenar, fato que contribui para reforçar a falsa hipótese de inocuidade e garantia de segurança das plantas medicinais quando comparadas a medicamentos alopáticos (MENGUE et al., 2001; ERNEST, 2002; VEIGA JR. et al., 2005).

Com relação a toxicidade referente ao potencial teratogênico e abortivo, tem-se espécies vegetais de uso contra indicado durante a gestação, como por exemplo as espécies: arruda (Ruta graveolens) (GUTIÉRREZ-PAJARES et al., 2003; FREITAS et al., 2005), boldo (Peumus boldus) (ALMEIDA \& LEMONICA, 2000), buchinha (Luffa operculata) (NGAl et al., 1992; NGAl et al., 1993), confrei (Symphytum officinale) (MENGUE et al., 2001; 
BELEW, 1999), losna (Artemisia absinthium) (MENGUE et al., 2001), melão-de-sãocaetano (Momordica charantia) (MENGUE et al., 2001).

Segundo Campessato (2005), é fundamental conhecer as substâncias de origem vegetal que devem ser contra indicadas durante a gestação, com o objetivo de facilitar a tomada de medidas educativas entre profissionais da área de saúde e população, além de contribuir para a saúde dos pacientes e melhora da qualidade dos fitoterápicos nacionais.

Nesse sentido, destaca-se o Rio de Janeiro por ser o único estado brasileiro a ter uma legislação específica sobre o uso de plantas medicinais por mulheres gestantes (CLARKE et al., 2007). A Resolução da Secretaria de Estado de Saúde/RJ № 1757, visa informar sobre o uso de plantas medicinais por gestantes, contra indicando o uso interno de drogas vegetais medicinais, em geral durante $01^{\circ}$ trimestre de gestação e lactação, cujos estudos toxicológicos não estejam concluídos (BRASIL, 2002).

\section{DISCUSSÃO}

Considerando a riqueza da biodiversidade, e que as plantas medicinais possuem constituição química complexa, é possível que o perfil químico e farmacológico de uma determinada espécie utilizada popularmente com finalidade medicinal, não tenha sido completamente compreendido do ponto de vista cientifico. Portanto, observa-se a recomendação de cautela na administração destes produtos. Em geral, o fator cultural referente ao uso tradicional de plantas medicinais vem sendo utilizado como indicio de segurança, porém nesse sentido ainda tem-se pouca literatura sobre a avaliação da toxicidade reprodutiva.

Os estudos de toxicidade envolvem experimentos em animais, mas nem sempre esse modelo de toxicidade se reproduz no organismo humano. Em adição existem problemas relacionados a base de dados de toxicidade de plantas medicinais, porque grande parte dos relatos envolvendo intoxicação, acidental ou não, carece de informações a respeito de variáveis como a parte do vegetal utilizada, quantidade, e mesmo a correta identificação botânica, pois é comum que mais de uma espécie vegetal seja popularmente conhecida por um mesmo nome, por isso a necessidade da correta identificação pelo nome cientifico. Com relação as orientações sobre o uso, recomenda- se que gestantes e lactantes não utilizem qualquer medicamento sem o conhecimento prévio do seu médico. Nesse sentido, cabe aos profissionais da saúde informar e esclarecer a população sobre $o$ 
risco do uso de plantas medicinais e fitoterápicos na gravidez, principalmente no que se refere a os perigos da automedicação.

A respeito da toxicidade de plantas medicinais durante a gravidez e lactação observa-se também que os dados de literatura são escassos, e muitas vezes contraditórios. Os estudos sobre a toxicidade de plantas medicinais e fitoterápicos durante a gestação e lactação, quando comparados, apresentam diferenças inerentes aos métodos utilizamos na pesquisa. Dessa maneira, justifica-se a necessidade e a importância do investimento tecnológico e cientifico em pesquisas que abordam o potencial toxico de plantas medicinais utilizadas durante a gravidez e lactação.

\section{CONCLUSÃO}

A ideia, muitas vezes mistificada, de que o uso irrestrito de plantas medicinais não faz mal à saúde é equivocada, perigosa e portanto, merece atenção de órgãos reguladores, profissionais da área da saúde e comunidade acadêmica com o objetivo de informar e contribuir para uma melhor prática e emprego das plantas medicinais e produtos fitoterápicos não só quando utilizados por mulheres gestantes e sim visando a saúde da população de uma maneira ampla.

\section{REFERÊNCIAS}

ALMEIDA, F.C.G.; LEMONICA, I.P. The toxicity of Coleus barbatus B. on the different periods of pregnancy in rats. J Ethnopharmacol. v.73, n.1-2, p.53-60, 2000.

AFZAL, M., AL-HADIDI, D., MENON, M., PESEK, J., DHAMI, M.S. Ginger: an ethnomedical, chemical and pharmacological review. Drug Metab. Drug Interact, v.18, p.159-190, 2001.

BADGUJAR, S.B.; PATEL, V.V.; BANDIVDEKAR, A.H. Foeniculum vulgare Mill: a review of its botany, phytochemistry, pharmacology, contemporary application, and toxicology. BioMed. Res. Int. p.1-32, 2014.

BAHMANI, M.; SHIRZAD, H.; MIRHOSSEINI, M.; MESRIPOUR, A.; RAFIEIAN-KOPAEI, M. A review on ethnobotanical and therapeutic uses of fenugreek (Trigonella foenum-graceum 
L), Journal of Evidence-Based Complementary and Alternative Medicine, v.21,n.1,p. 53-62, 2016.

BARILLI, S.L.S.; PEREIRA M.S.L; FOSCARINI P.T; DA SILVA F.C; MONTANARI T. An experimental investigation on effect of Foeniculum vulgare Mill. on gestation. Reprodução \& Climatério. v.27, n.2, p.73- 80, 2012.

BARON, T.H.; RAMÍREZ, B.; RICHTER, J.E. Gastrointestinal Motility Disorders During Pregnancy. Ann Intern Med., v.118, n.5, p.366-75, 1993.

BELEW, C. Herbs and Childbearing woman. J. Nurse Midwifery., v.44, n.3, p.231-252, 1999.

BETZOLD, C.M. Galactagogues. J. Midwifery Women Health, v.49, p.151-154, 2004.

BRASIL. Ministério da Saúde. Secretária de Vigilância Sanitária. Portaria n 116, de 08 de agosto de 1996. Diário Oficial da União, 12 de agosto de 1996.

BRASIL. Secretaria de Saúde do Estado do Rio de Janeiro. Resolução nำ 1757, de 18 de fevereiro de 2002. Diário Oficial do Estado do Rio de Janeiro, 20 de fevereiro de 2002.

BRASIL. Ministério da Saúde. ANVISA. Portaria $n^{\circ}$ 48, de 16 de março de 2004. Diário Oficial da União, DF, 18 de março de.2004.

BRASIL. Ministério da Saúde. Portaria no. 971, de 3 de maio de 2006. Aprova a Política Nacional de Práticas Integrativas e Complementares no SUS. Diário Oficial da União, Brasília, DF, 4 de maio de 2006. 2006a.

BRASIL. Ministério da Saúde. Decreto nํ⒌813, de 22 de junho de 2006. Aprova a Política Nacional de Plantas Medicinais e Fitoterápicos e dá outras providências. Diário Oficial da União, Brasília, DF, 23 de junho de 2006. 2006b.

CAMINHA, M.F.C.; SERVA, V.B.; ARRUDA, I.K.; FILHO, M.B. Aspectos históricos, socioeconômicos e institucionais do aleitamento materno. Revista Brasileira de Saúde Materno infantil, v.10, n.1, p.25-37, 2010. 
CAMPESATO, V.R. Uso de plantas medicinais durante a gravidez e risco para malformações congênitas [Tese]. Porto Alegre (RS): Universidade Federal do Rio Grande do Sul; 2005.

CHEZ, R.A.; JONAS, W.B. Complementary and alternative medicine. Part I:clinical studies in obstetrics. Obstet Gynecol Surv., v.52, p.704-708, 1997.

CHRUBASIK, S., PITTLER, M.H., ROUFOGALIS, B.D. Zingiberis rhizoma: a comprehensive review on the ginger effect and efficacy profiles. Phytomedicine, v.12, p.684701, 2005.

CONOVER, E.A. Herbal agents and over-the-counter medications in pregnancy. Best Pract Res Clin Endocrinol Metab., v.17, n.2, p.237-251, 2003.

DI STASI, L.C. (Org.). Plantas medicinais: arte e ciência. Um guia de estudo interdisciplinar. São Paulo: Editora da Universidade Estadual Paulista, 1996. 230 p., il. (Natura Naturata).

ERNST, E. Herbal medicinal products during pregnancy: are they safe? BJOG., v.109, p.227-235, 2002. FALEIROS, F.T.; TREZZA, E.M.; CARANDINA, L. Factors influencing breastfeeding decision and duration. Rev Nutr., v.19, p.623-630, 2006.

FALKENBERG, M.B. Quinonas. In: SIMÕES, C. M. O. (Org.) et al. Farmacognosia: da planta ao medicamento. 6.ed. Porto Alegre: Editora da UFRGS, 2010. p. 657-683.

FICKER, C., SMITH, M.L., AKPAGANA, K., GBEASSOR, M., ZHANG, J., DURST, T., ASSABGUI, R., ARNASON, J.T. Bioassay-guided isolation and identification of antifungal compounds from ginger. Phytother. Res., v.17, p.897-902, 2003.

FLYNN, D.L., RAFFERTY, M.F., BOCTOR, A.M. Inhibition of human neutrophil 5lipoxygenase activity by gingerdione, shogaol, capsaicin and related pungent compounds. Prostaglandins Leukot. Med., v.24, p.195-198, 1968.

FREITAS, T.G.; AUGUSTO, P.M.; MONTANARI, T. Effect of Ruta graveolens L. on pregnant mice. Contraception. v.71, n.1, p.74-7, 2005. 
GARTNER, L.M.; MORTON, J.; LAWRENCE, R.A. Breastfeeding and the use of human milk. Pediatrics, v.115, n.2, p.496-506, 2005.

GOODWIN, T.M. Nausea and vomiting of pregnancy: an obstetric syndrome. American Journal of Obstetrics and Gynecology, v.186, 2002.

GOYAL, S.; GUPTA, N.; CHATTERJEE, S. Investigating Therapeutic Potential of Trigonella foenum- graecum L. as Our Defense Mechanism against Several Human Diseases. J. Toxicol., p.1-10, 2016. GRZANNA, R., LINDMARK, L., FRONDOZA, C.G. Ginger - an herbal medicinal product with broad anti- inflammatory actions. J. Med. Food, v.8, p.125-132, 2005.

GUTIÉRREZ-PAJARES, J.L.; ZÚÑIGA, L.; PINO, J. Ruta graveolens aqueous extract retards mouse preimplantation embryo development. Reproduct Toxicol. v.17, n.6, p.66772, 2003.

HEITMANN K., NORDENG H., HOLST L. Safety of ginger use in pregnancy: results from a large population- based cohort study. Eur J Clin Pharmacol., v.69,n.2, p.269-77, 2013.

IQBAL, Z., LATEEF, M., AKHTAR, M.S., GHAYUR, M.N., GILANI, A.H. In vivo anthelmintic activity of ginger against gastrointestinal nematodes of sheep. J. Ethnopharmacol., v.106, p.285-287, 2006.

JEWELL, D.J; YOUNG, G. Interventions for treating constipation in pregnancy. Cochrane Database Syst Rev., 2001.

LETE I, ALLUÉ J. The effectiveness of ginger in the prevention of nausea and vomiting during pregnancy and chemotherapy. Integr Med Insights., v.11, p.11-17, 2016.

LOUIK, C.; GARDINER, P.; KELLEY, K.; MITCHELL, A.A. Use of herbal treatments in pregnancy. Am J Obstet Gynecol., v.202, n.5, 2010.

MCNANAMAN, J.L.; NEVILLE, M.C. Mammary physiology and milk secretion. Adv Drug Deliv Rev., v.55, p.629-641,2003. 
MENGUE, S.S.; MENTZ, L.A.; SCHENKEL, E.P. Uso de plantas medicinais na gravidez. In: Sanseverino, V.T.M.; Spreitzer, T.D.; Schüler-Faccini L. Manual de Teratogênese. Porto Alegre: Editora da Universidade/UFRGS, pp. 423-447. 2001

MIGUEL, M.D.; MIGUEL, O.G. 2000. Desenvolvimento de fitoterápicos. São Paulo: Robe Editorial. 115p. MILLER, F. Nausea and vomiting in pregnancy: the problem of perception-is it really a disease? American Journal of Obstetrics and Gynecology, v.186, n.5, p.182183, 2002.

NEWCOMB, P.A.; STORER, B.E.; LONGNECKER, M.P.; MITTENDORF, R.; GREENBERG, E.R.; CLAPP, R.W.; BURKE, K.P.; WILLETT, W.C.; MACMAHON, B. Lactation and a reduced risk of premenopausal breast cancer. N Engl J Med., v.330, n.2, p.81-87, 1994.

NGAI, T.B.; WONG, R.N.; YEUNG, H.W. Two proteins with ribosome-inactivating, cytotoxic and abortifacient activities from seeds of Luffa cylindrica roem (Cucurbitaceae). Biochem Int. v.27, n.2, p.197- 207, 1992.

NGAI, T.B.; CHAN, W.Y.; YEUNG, H.W. The ribosome-inactivating, antiproliferative and teratogenic activities and immunoreactivities of a protein from seeds of Luffa aegyptiaca (Cucurbitaceae). Gen Pharmacol. v.24, n.3, p.655-8, 1993.

OJEWOLE, J.A., Analgesic, antiinflammatory and hypoglycaemic effects of ethanol extract of Zingiber officinale (Roscoe) rhizomes (Zingiberaceae) in mice and rats. Phytother. Res., v.20, p.764-772, 2006.

OLIVEIRA, A.C.D.; ROPKE, C. Os dez anos da Política Nacional de Plantas Medicinais e Fitoterápicos (PNPMF) e os principais entraves da cadeia produtiva de extratos vegetais e medicamentos fitoterápicos no Brasil. 2016. Rev Fitos., v.10, n.2, p.185-198, 2016.

OSHIRO, M.C.; MIGUEL, M.D.; DIAS, J.F.G.; GOMES, E.C.; MIGUEL, O.G. A evolução do registro e prescrição de fitoterápicos no Brasil sob perspectiva legal e sanitária. Vigil sanit debate., v.4, n.4, p.116-122, 2016. 
OUZIR, M., EL-BAIRI, K., AMZAZI, S., Toxicological properties of fenugreek (Trigonella foenum-graecum). Food Chem. Toxicol., v.96, p.145-154, 2016.

Palmer AK. Regulatory requirements for reproductive toxicology: theory and practice. In: Kimmel C, Buelke- Sam J, editors. Developmental Toxicology, 1st ed. New York: Raven Press, 1981. pp. 259-87.

PORTNOI G, CHNG L-A, KARIMI-TABESH L, KOREN G, TAN MP, EINARSON A. Prospective comparative study of the safety and effectiveness of ginger for the treatment of nausea and vomiting in pregnancy. Am J Obstet Gynecol., v.89, n.5, p.1374-7, 2003.

RAHIMI, R.; ARDEKANI, M.R.S. Medicinal properties of Foeniculum vulgare Mill. in traditional Iranian medicine and modern phytotherapy. Chinese Journal of Integrative Medicine, v.19, n. 1, p. 73-79, 2013.

REA, M.F. Os benefícios da amamentação para a saúde da mulher. J Pediatr., v. 80, n.5, 2004.

RUEDY, J. Teratogenic Risk of Drugs Used in Early Pregnancy. Can. Fam. Physician.,v.30, p.2133-2136, 1984

SHULZ, V.; HÄNSEL, R.; TYLER, V.E. Fitoterapia Racional: Um Guia de Fitoterapia para as Ciências da Saúde. Barueri: Manole; 2002. 386p.

TABARES, F.P.; JARAMILLO, J.V.B.; RUIZ-CORTÉS, Z.T. Pharmacological Overview of Galactogogues. Veterinary Medicine International, p.1-20, 2014.

TALOUBI, L.M.; RHOUDA, H.; BELAHCEN, A.; SMIRES, N., THIMOU, A., MDAGHRI, A.A. An overview of plants causing Teratogenicity: fenugreek (Trigonella foenum graecum). Int. J. Pharm. Sci. Res., v.4, n.2, p.516-519, 2013.

THOMSON M, CORBIN R, LEUNG L. Effects of ginger for nausea and vomiting in early pregnancy: a meta- analysis. J Am Board Fam Med., v.27, n.1, p.115-22, 2014. 
THOMSON, M., AL-QATTAN, K.K., AL-SAWAN, S.M., ALNAQEEB, M.A., KHAN, I., ALI, M. The use of ginger (Zingiber officinale Rosc.) as a potential anti-inflammatory and antithrombotic agent. Prostaglandins Leukot. Essent. Fatty Acid, v.67, p.475-478, 2002.

TOMA, T.S.; REA, M.F. Benefícios da amamentação para a saúde da mulher e da criança: um ensaio sobre as evidências. Cad Saúde Pública., v.24, n.2,p.235-246, 2008.

VEIGA JR, V.F.; PINTO, A.C. Plantas medicinais: cura segura? Quím. Nova., v.28, n.3, p.519-528, 2005.

VENKATA, K.C.N.; SWAROOP, A.; BAGCHI, D.; BISHAYEE, A. A small plant with big benefits: Fenugreek (Trigonella foenum-graecum Linn.) for disease prevention and health promotion. Mol. Nutr. Food Res., v.61, n.6, 2017.

VILJOEN, E., VISSER, J., KOEN, N., MUSEKIWA, A. A systematic review and metaanalysis of the effect and safety of ginger in the treatment of pregnancy-associated nausea and vomiting. Nutr. J., v.13, n.20, 2014.

WOOLHOUSE, M. Complementary medicine for pregnancy complications. Australian Family Physician. v.35, n.9, 695, 2007.

ZAPANTIS A., STEINBERG J.G., SCHILIT L. Use of herbals as galactagogues. J Pharm Pract., v.25, n.2, p.222231, 2012. 